\title{
APPLICATION OF TOPSIS METHOD TO ANALYZE CAUSES OF SUICIDE THOUGHT IN DOMESTIC VIOLENCE
}

\author{
M.Clement Joe Anand, A.Victor Devadoss \\ Department of Mathematics, Loyola College, Chennai-34, India \\ arjoemi@gmail.com
}

\begin{abstract}
Technique for Order Preference by Similarity to Ideal Solution (TOPSIS) is the best method to find out the ideal solution of the problem. In recent years, TOPSIS has been widely applied to many fields with good results such as marketing strategy selection, mechatronic system optimization, network security management, location planning for urban distribution centers, business competition evaluation, irrigation water pricing decision making, emergency management, low carbon development evaluation, spaceflight mission planning, Environmental Science. This is the first time we study the causes of suicidal thought in domestic violence using TOPSIS Method. This paper has four sections. Section one gives the introduction to the suicide thought. Second section gives the Algorithm of TOPSIS method is explained. In section three we derive the framework of the research topic. Final section gives the conclusion based on our study.
\end{abstract}

Keywords: Domestic Violence, Intimate Partner, Suicide and TOPSIS.

\section{Introduction}

Suicide rates in India have increased steadily over the last few decades. The WHO estimates that there were $1,90,000$ suicide deaths in India in 2010 alone. Suicide has become the second leading cause of death among young Indians. It is the cause of twice as many deaths as HIV/AIDS and almost the same number as maternal deaths in young women [3].The large majority of suicide victims in India are married and the broad class of 'family problems' is the main reported cause of suicide for both men and women. Durkheim's ground breaking work, the relationship between marriage and suicides has been much studied empirically in sociology but mostly ignored in economics [14]. It emphasize how increased opportunities for women can accentuate tensions and marital discord within households, by challenging traditional roles, increasing the importance of negotiation and raising the potential for conflict. There are numerous empirical accounts investigating the possible consequences of female empowerment on suicides. Suicides of married women and men as a response to family conflict are a common occurrence particularly in developing countries. Canetto discusses the cultural ramifications of suicide-that relative to developed countries, where suicidal behavior tends to be interpreted as a symptom of individual mental health, in poorer countries, suicide is often considered a normal, albeit last resort response, to a serious family conflict.

There is no one single factor to account for violence perpetrated against women. Increasingly, research has focused on the interrelatedness of various factors that should improve our understanding of the problem within different cultural contexts. Several complex and interconnected institutionalized social and cultural factors have kept women particularly vulnerable to the violence directed at them, all of them manifestations of historically unequal power relations between men and women. Factors contributing to these unequal power relations include: socio economic forces, the family institution where power relations are enforced, fear of and control over female sexuality, belief in the inherent superiority of males, and legislation and cultural sanctions that have traditionally denied women and children an independent legal and social status. 


\section{The Topsis Method}

TOPSIS method is a technique for order preference by similarity to ideal solution and proposed by Hwang and Yoon (1981). The ideal solution (also called positive ideal solution) is a solution that maximizes the benefit criteria/attributes and minimizes the cost criteria/ attributes, whereas the negative ideal solution (also called anti-ideal solution) maximizes the cost criteria/ attributes and minimizes the benefit criteria/attributes. The so-called benefit criteria/attributes are those for maximization, while the cost criteria/attributes are those for minimization. The best alternative is the one, which is closest to the ideal solution and farthest from the negative ideal solution. In recent years, TOPSIS has been widely applied to many fields with good results such as marketing strategy selection[7], mechatronic system optimization [8], network security management [20], location planning for urban distribution centers[1], business competition evaluation[11], irrigation water pricing decision making[9], emergency management[21], low carbon development evaluation[12], spaceflight mission planning[18], Environmental studies and this is the first time we studying the causes of suicidal thought in domestic violence using TOPSIS Method. The basic steps of TOPSIS method are presented as follows.

Step 1: Construct a multi-criteria decision matrix.

Suppose a decision-making problem has $m$ alternatives, each of which consists of $n$ attributes. The $j^{\text {th }}$ criterion of the $i^{\text {th }}$ alternative is denoted as $x_{i j}(i=1,2, \ldots, m ; j=1,2, \ldots n)$, then the decision matrix $X$ of this problem is:

$$
X=\left(x_{i j}\right)_{m \times n}=\left[\begin{array}{cccc}
x_{11} & x_{12} & \cdots & x_{1 n} \\
x_{21} & x_{22} & \cdots & x_{2 n} \\
\vdots & \vdots & \vdots & \vdots \\
x_{m 1} & x_{m 2} & \cdots & x_{m n}
\end{array}\right]
$$

According to the relationship between values of the criteria and their relative superiority, we can divide the criteria into benefit-type and cost-type. The more the value is, the better the criterion is, which is called a benefit-type criterion; in contrast, the more the value is, the worse criterion is, which is called a cost-type criterion.

Step 2: Calculate the normalized decision matrix. In order to make the different criteria comparable, the decision matrix $X$ is needed to be normalized, resulting in the Normalize the decision matrix $X=\left(x_{i j}\right)_{m \times n}$ using the equation below:

$$
r_{i j}=\frac{x_{i j}}{\sqrt{\sum_{k=1}^{m} x_{k j}^{2}}}, \quad i=1,2, \ldots, m ; \quad j=1,2, \ldots, n
$$

Where $r_{i j}$ is the normalized criteria/attribute value /rating.

Step 3: Calculate the weighted normalized decision matrix.

The value $v_{i j}$ is calculated as $v_{i j}=w_{j} r_{i j}$, $i=1,2, \ldots, m ; j=1,2, \ldots, n$, Where $w_{j}$ is the relative weight of the $j^{\text {th }}$ criterion or attribute and $\sum_{j=1}^{n} w_{j}=1$. Thus, the weighted normalized decision matrix $V=\left(v_{i j}\right)_{m \times n}$ is established.

Step 4: Determine the Positive-ideal and Negative-ideal solutions.

$$
\begin{aligned}
& \text { Given } A^{+}=\left\{v_{1}^{+}, v_{2}^{+}, \ldots, v_{m}^{+}\right\} \\
& =\left\{\left(\max _{j} v_{i j} \mid j \in \Omega_{b}\right),\left(\min _{j} v_{i j} \mid j \in \Omega_{c}\right)\right\} \\
& A^{-}=\left\{v_{1}^{-}, v_{2}^{-}, \ldots, v_{m}^{-}\right\} \\
& =\left\{\left(\min _{j} v_{i j} \mid j \in \Omega_{b}\right),\left(\max _{j} v_{i j} \mid j \in \Omega_{c}\right)\right\},
\end{aligned}
$$

where $\Omega_{b}$ and $\Omega_{c}$ are the sets of benefit criteria/attributes and cost criteria/attributes, respectively. It is easy to see that applying Range Method to standardize the data can help to determine the numerical values of the positive ideal and negative ideal solution quickly.

Step 5: Calculate the separation measures. The separation of each alternative from the positive ideal solution, $d_{i}^{+}$is given as 


$$
d_{i}^{+}=\sqrt{\sum_{j=1}^{n}\left(v_{i j}-v_{j}^{+}\right)^{2}}, i=1,2, \ldots, m ;
$$

Similarly, the separation of each alternative from the negative ideal solution, $d_{i}^{-}$is given as

$$
d_{i}^{-}=\sqrt{\sum_{j=1}^{n}\left(v_{i j}-v_{j}^{-}\right)^{2}}, i=1,2, \ldots, m ;
$$

Step 6: Calculate the relative closeness to the ideal solution.

The relative closeness of the $i^{\text {th }}$ alternative with respect to the ideal solution $C_{i}$ is defined as

$$
D_{i}=\frac{d_{i}^{-}}{d_{i}^{+}+d_{i}^{-}}, i=1,2, \ldots, m ;
$$

The relative closeness of the $d_{i}^{+} \geq 0$ and $d_{i}^{-} \geq 0$, then clearly, $D_{i} \in[0,1]$.

Step 7: Rank the preference order.Rank the alternatives according to the relative closeness to the ideal solution. The best alternative is the one with the greatest relative closeness to the ideal solution.

\section{Research Framework}

The research follows the following steps. First we interviewed 100 women's in Chennai. From that identified the causes of suicidal thought in the domestic violence. The theme of our research i.e., domestic violence against women is happening by following criteria:

$\mathrm{C}_{1}$ - Husband's Mother(Mother-in-law)

$\mathrm{C}_{2}$ - Husband's Father(Father-in-law)

$\mathrm{C}_{3}$ - Intimate partner (Husband)

We are meant to construct the hierarchical structure of the research.

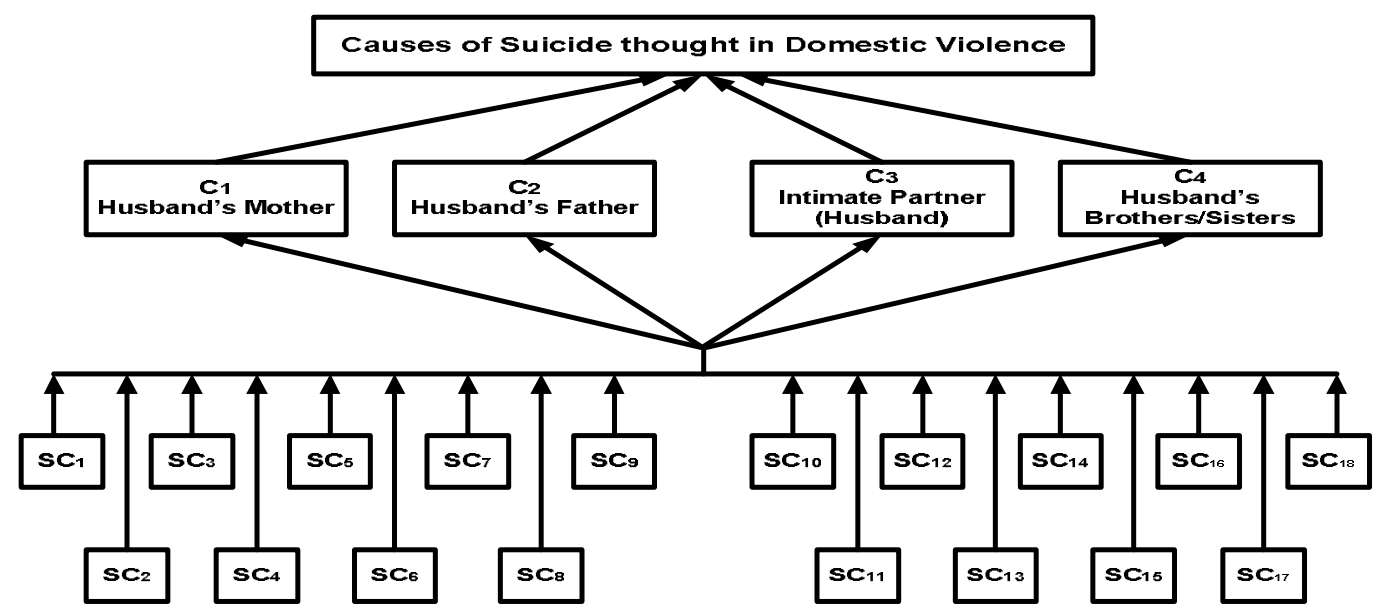

Integrated Intelligent Research (IIR) 
Step 1: A multi-criteria decision matrix is

Weight $\quad 0.3$

0.2

0.4

0.1

\begin{tabular}{|c|c|c|c|c|}
\hline Attributes & $\mathrm{C}_{1}$ & $\mathrm{C}_{2}$ & $\mathrm{C}_{3}$ & $\mathrm{C}_{4}$ \\
\hline $\mathbf{S C}_{1}$ & 9 & 7 & 9 & 6 \\
\hline $\mathrm{SC}_{2}$ & 8 & 6 & 8 & 3 \\
\hline $\mathrm{SC}_{3}$ & 8 & 5 & 6 & 1 \\
\hline $\mathbf{S C}_{4}$ & 6 & 4 & 7 & 2 \\
\hline $\mathrm{SC}_{5}$ & 8 & 6 & 9 & 4 \\
\hline $\mathrm{SC}_{6}$ & 9 & 7 & 9 & 7 \\
\hline $\mathbf{S C}_{7}$ & 6 & 4 & 8 & 2 \\
\hline $\mathrm{SC}_{8}$ & 9 & 3 & 9 & 8 \\
\hline $\mathrm{SC}_{9}$ & 6 & 2 & 6 & 4 \\
\hline $\mathrm{SC}_{10}$ & 4 & 6 & 7 & 5 \\
\hline $\mathrm{SC}_{11}$ & 9 & 8 & 9 & 6 \\
\hline $\mathrm{SC}_{12}$ & 5 & 4 & 5 & 1 \\
\hline $\mathrm{SC}_{13}$ & 9 & 3 & 7 & 8 \\
\hline $\mathrm{SC}_{14}$ & 8 & 4 & 9 & 4 \\
\hline $\mathrm{SC}_{15}$ & 8 & 5 & 8 & 2 \\
\hline $\mathrm{SC}_{16}$ & 7 & 3 & 9 & 3 \\
\hline $\mathbf{S C}_{17}$ & 7 & 2 & 9 & 2 \\
\hline $\mathrm{SC}_{18}$ & 8 & 4 & 8 & 4 \\
\hline
\end{tabular}

Step 2:

The normalized decision matrix is

\begin{tabular}{|c|c|c|c|c|}
\hline Attributes & $\mathbf{C}_{\mathbf{1}}$ & $\mathbf{C}_{\mathbf{2}}$ & $\mathbf{C}_{\mathbf{3}}$ & $\mathbf{C}_{\mathbf{4}}$ \\
\hline $\mathbf{S C}_{\mathbf{1}}$ & 0.2796 & 0.3356 & 0.2656 & 0.3103 \\
\hline $\mathbf{S C}_{\mathbf{2}}$ & 0.2485 & 0.2877 & 0.2361 & 0.1551 \\
\hline $\mathbf{S C}_{\mathbf{3}}$ & 0.2485 & 0.2397 & 0.1771 & 0.0517 \\
\hline $\mathbf{S C}_{\mathbf{4}}$ & 0.1864 & 0.1918 & 0.2066 & 0.1034 \\
\hline $\mathbf{S C}_{\mathbf{5}}$ & 0.2485 & 0.2877 & 0.2656 & 0.2068 \\
\hline $\mathbf{S C}_{\mathbf{6}}$ & 0.2796 & 0.3356 & 0.2656 & 0.3620 \\
\hline $\mathbf{S C}_{\mathbf{7}}$ & 0.1864 & 0.1918 & 0.2361 & 0.1034 \\
\hline $\mathbf{S C}_{\mathbf{8}}$ & 0.2796 & 0.1438 & 0.2656 & 0.4137 \\
\hline $\mathbf{S C}_{\mathbf{9}}$ & 0.1864 & 0.0959 & 0.1771 & 0.2068 \\
\hline $\mathbf{S C}_{\mathbf{1 0}}$ & 0.1243 & 0.2877 & 0.2066 & 0.2585 \\
\hline $\mathbf{S C}_{\mathbf{1 1}}$ & 0.2796 & 0.3836 & 0.2656 & 0.3103 \\
\hline $\mathbf{S C}_{\mathbf{1 2}}$ & 0.1553 & 0.1918 & 0.1476 & 0.0517 \\
\hline $\mathbf{S C}_{\mathbf{1 3}}$ & 0.2796 & 0.1438 & 0.2066 & 0.4137 \\
\hline $\mathbf{S C}_{\mathbf{1 4}}$ & 0.2485 & 0.1918 & 0.2656 & 0.2068 \\
\hline $\mathbf{S C}_{\mathbf{1 5}}$ & 0.2485 & 0.2397 & 0.2361 & 0.1034 \\
\hline $\mathbf{S C}_{\mathbf{1 6}}$ & 0.2175 & 0.1438 & 0.2656 & 0.1551 \\
\hline $\mathbf{S C}_{\mathbf{1 7}}$ & 0.2175 & 0.0959 & 0.2656 & 0.1034 \\
\hline $\mathbf{S C}_{\mathbf{1 8}}$ & 0.2485 & 0.1918 & 0.2361 & 0.2068 \\
\hline & & & & \\
\hline & & & & \\
\hline
\end{tabular}


Step 3: Multiply each column by $w_{j}$ to get $v_{i j}$.

\begin{tabular}{|c|c|c|c|c|}
\hline Attributes & $\mathbf{C}_{\mathbf{1}}$ & $\mathbf{C}_{\mathbf{2}}$ & $\mathbf{C}_{\mathbf{3}}$ & $\mathbf{C}_{\mathbf{4}}$ \\
\hline $\mathbf{S C}_{\mathbf{1}}$ & 0.0839 & 0.0671 & 0.1063 & 0.0310 \\
\hline $\mathbf{S C}_{\mathbf{2}}$ & 0.0746 & 0.0575 & 0.0944 & 0.0155 \\
\hline $\mathbf{S C}_{\mathbf{3}}$ & 0.0746 & 0.0479 & 0.0708 & 0.0052 \\
\hline $\mathbf{S C}_{\mathbf{4}}$ & 0.0559 & 0.0384 & 0.0826 & 0.0103 \\
\hline $\mathbf{S C}_{\mathbf{5}}$ & 0.0746 & 0.0575 & 0.1063 & 0.0207 \\
\hline $\mathbf{S C}_{\mathbf{6}}$ & 0.0839 & 0.0671 & 0.1063 & 0.0362 \\
\hline $\mathbf{S C}_{\mathbf{7}}$ & 0.0559 & 0.0384 & 0.0944 & 0.0103 \\
\hline $\mathbf{S C}_{\mathbf{8}}$ & 0.0839 & 0.0288 & 0.1063 & 0.0414 \\
\hline $\mathbf{S C}_{\mathbf{9}}$ & 0.0559 & 0.0192 & 0.0708 & 0.0207 \\
\hline $\mathbf{S C}_{\mathbf{1 0}}$ & 0.0373 & 0.0575 & 0.0826 & 0.0259 \\
\hline $\mathbf{S C}_{\mathbf{1 1}}$ & 0.0839 & 0.0767 & 0.1063 & 0.0310 \\
\hline $\mathbf{S C}_{\mathbf{1 2}}$ & 0.0466 & 0.0384 & 0.0590 & 0.0052 \\
\hline $\mathbf{S C}_{\mathbf{1 3}}$ & 0.0839 & 0.0288 & 0.0826 & 0.0414 \\
\hline $\mathbf{S C}_{\mathbf{1 4}}$ & 0.0746 & 0.0384 & 0.1063 & 0.0207 \\
\hline $\mathbf{S C}_{\mathbf{1 5}}$ & 0.0746 & 0.0479 & 0.0944 & 0.0103 \\
\hline $\mathbf{S C}_{\mathbf{1 6}}$ & 0.0652 & 0.0288 & 0.1063 & 0.0155 \\
\hline $\mathbf{S C}_{\mathbf{1 7}}$ & 0.0652 & 0.0192 & 0.1063 & 0.0103 \\
\hline $\mathbf{S C}_{\mathbf{1 8}}$ & 0.0746 & 0.0384 & 0.0944 & 0.0207 \\
\hline & & & & \\
\hline
\end{tabular}

Step 4(a): Positive Ideal Solution is

$A^{+}=\{0.0839,0.0767,0.1063,0.0414\}$

\begin{tabular}{|c|c|c|c|c|}
\hline Attributes & $\mathbf{C}_{\mathbf{1}}$ & $\mathbf{C}_{\mathbf{2}}$ & $\mathbf{C}_{\mathbf{3}}$ & $\mathbf{C}_{\mathbf{4}}$ \\
\hline $\mathbf{S C}_{\mathbf{1}}$ & 0.0839 & 0.0671 & 0.1063 & 0.0310 \\
\hline $\mathbf{S C}_{\mathbf{2}}$ & 0.0746 & 0.0575 & 0.0944 & 0.0155 \\
\hline $\mathbf{S C}_{\mathbf{3}}$ & 0.0746 & 0.0479 & 0.0708 & 0.0052 \\
\hline $\mathbf{S C}_{\mathbf{4}}$ & 0.0559 & 0.0384 & 0.0826 & 0.0103 \\
\hline $\mathbf{S C}_{\mathbf{5}}$ & 0.0746 & 0.0575 & 0.1063 & 0.0207 \\
\hline $\mathbf{S C}_{\mathbf{6}}$ & 0.0839 & 0.0671 & 0.1063 & 0.0362 \\
\hline $\mathbf{S C}_{\mathbf{7}}$ & 0.0559 & 0.0384 & 0.0944 & 0.0103 \\
\hline $\mathbf{S C}_{\mathbf{8}}$ & 0.0839 & 0.0288 & 0.1063 & 0.0414 \\
\hline $\mathbf{S C}_{\mathbf{9}}$ & 0.0559 & 0.0192 & 0.0708 & 0.0207 \\
\hline $\mathbf{S C}_{\mathbf{1 0}}$ & 0.0373 & 0.0575 & 0.0826 & 0.0259 \\
\hline $\mathbf{S C}_{\mathbf{1 1}}$ & 0.0839 & 0.0767 & 0.1063 & 0.0310 \\
\hline $\mathbf{S C}_{\mathbf{1 2}}$ & 0.0466 & 0.0384 & 0.0590 & 0.0052 \\
\hline $\mathbf{S C}_{\mathbf{1 3}}$ & 0.0839 & 0.0288 & 0.0826 & 0.0414 \\
\hline $\mathbf{S C}_{\mathbf{1 4}}$ & 0.0746 & 0.0384 & 0.1063 & 0.0207 \\
\hline $\mathbf{S C}_{\mathbf{1 5}}$ & 0.0746 & 0.0479 & 0.0944 & 0.0103 \\
\hline
\end{tabular}




\begin{tabular}{|l|l|l|l|l|}
\hline $\mathbf{S C}_{\mathbf{1 6}}$ & 0.0652 & 0.0288 & 0.1063 & 0.0155 \\
\hline $\mathbf{S C}_{\mathbf{1 7}}$ & 0.0652 & 0.0192 & 0.1063 & 0.0103 \\
\hline $\mathbf{S C}_{\mathbf{1 8}}$ & 0.0746 & 0.0384 & 0.0944 & 0.0207 \\
\hline
\end{tabular}

Step 4(b): Negative Ideal Solution is

$A^{-}=\{0.0373,0.0192,0.0590,0.0052\}$

\begin{tabular}{|c|c|c|c|c|}
\hline Attributes & $\mathbf{C}_{\mathbf{1}}$ & $\mathbf{C}_{\mathbf{2}}$ & $\mathbf{C}_{\mathbf{3}}$ & $\mathbf{C}_{\mathbf{4}}$ \\
\hline $\mathbf{S C}_{\mathbf{1}}$ & 0.0839 & 0.0671 & 0.1063 & 0.0310 \\
\hline $\mathbf{S C}_{\mathbf{2}}$ & 0.0746 & 0.0575 & 0.0944 & 0.0155 \\
\hline $\mathbf{S C}_{\mathbf{3}}$ & 0.0746 & 0.0479 & 0.0708 & 0.0052 \\
\hline $\mathbf{S C}_{\mathbf{4}}$ & 0.0559 & 0.0384 & 0.0826 & 0.0103 \\
\hline $\mathbf{S C}_{\mathbf{5}}$ & 0.0746 & 0.0575 & 0.1063 & 0.0207 \\
\hline $\mathbf{S C}_{\mathbf{6}}$ & 0.0839 & 0.0671 & 0.1063 & 0.0362 \\
\hline $\mathbf{S C}_{\mathbf{7}}$ & 0.0559 & 0.0384 & 0.0944 & 0.0103 \\
\hline $\mathbf{S C}_{\mathbf{8}}$ & 0.0839 & 0.0288 & 0.1063 & 0.0414 \\
\hline $\mathbf{S C}_{\mathbf{9}}$ & 0.0559 & 0.0192 & 0.0708 & 0.0207 \\
\hline $\mathbf{S C}_{\mathbf{1 0}}$ & 0.0373 & 0.0575 & 0.0826 & 0.0259 \\
\hline $\mathbf{S C}_{\mathbf{1 1}}$ & 0.0839 & 0.0767 & 0.1063 & 0.0310 \\
\hline $\mathbf{S C}_{\mathbf{1 2}}$ & 0.0466 & 0.0384 & 0.0590 & 0.0052 \\
\hline $\mathbf{S C}_{\mathbf{1 3}}$ & 0.0839 & 0.0288 & 0.0826 & 0.0414 \\
\hline $\mathbf{S C}_{\mathbf{1 4}}$ & 0.0746 & 0.0384 & 0.1063 & 0.0207 \\
\hline $\mathbf{S C}_{\mathbf{1 5}}$ & 0.0746 & 0.0479 & 0.0944 & 0.0103 \\
\hline $\mathbf{S C}_{\mathbf{1 6}}$ & 0.0652 & 0.0288 & 0.1063 & 0.0155 \\
\hline $\mathbf{S C}_{\mathbf{1 7}}$ & 0.0652 & 0.0192 & 0.1063 & 0.0103 \\
\hline $\mathbf{S C}_{\mathbf{1 8}}$ & 0.0746 & 0.0384 & 0.0944 & 0.0207 \\
\hline & & & & \\
\hline
\end{tabular}

This Step 4(a) and 4(b) is giving the positive ideal (maximum criteria) and negative ideal (minimum criteria) solution of the attributes Mother- in-law, Father-in-law, Intimate partner, Brothers-Sister-inlaw.

Step 5(a): The separation of each alternative from the positive ideal solution, $d_{i}^{+}$is

\begin{tabular}{|c|c|c|c|c|c|c|c|c|}
\hline \multirow{2}{*}{$\begin{array}{c}\text { Attribute } \\
\mathrm{S}\end{array}$} & \multicolumn{2}{|c|}{$\mathbf{C}_{\mathbf{1}}$} & \multicolumn{2}{c|}{$\mathbf{C}_{\mathbf{2}}$} & \multicolumn{2}{c|}{$\mathbf{C}_{\mathbf{3}}$} & \multicolumn{2}{c|}{$\mathbf{C}_{\mathbf{4}}$} \\
\cline { 2 - 9 } & $v_{i j}-v_{j}^{+}$ & $\left(v_{i j}-v_{j}^{+}\right)^{2}$ & $v_{i j}-v_{j}^{+}$ & $\left(v_{i j}-v_{j}^{+}\right)^{2}$ & $v_{i j}-v_{j}^{+}$ & $\left(v_{i j}-v_{j}^{+}\right)^{2}$ & $v_{i j}-v_{j}^{+}$ & $\left(v_{i j}-v_{j}^{+}\right)^{2}$ \\
\hline $\mathbf{S C}_{\mathbf{1}}$ & 0.0000 & 0.0000 & -0.0096 & 0.0001 & 0.0000 & 0.0000 & -0.0103 & 0.0001 \\
\hline $\mathbf{S C}_{\mathbf{2}}$ & -0.0093 & 0.0001 & -0.0192 & 0.0004 & -0.0118 & 0.0001 & -0.0259 & 0.0007 \\
\hline $\mathbf{S C}_{\mathbf{3}}$ & -0.0093 & 0.0001 & -0.0288 & 0.0008 & -0.0354 & 0.0013 & -0.0362 & 0.0013 \\
\hline $\mathbf{S C}_{\mathbf{4}}$ & -0.0280 & 0.0008 & -0.0384 & 0.0015 & -0.0236 & 0.0006 & -0.0310 & 0.0010 \\
\hline $\mathbf{S C}_{\mathbf{5}}$ & -0.0093 & 0.0001 & -0.0192 & 0.0004 & 0.0000 & 0.0000 & -0.0207 & 0.0004 \\
\hline $\mathbf{S C}_{\mathbf{6}}$ & 0.0000 & 0.0000 & -0.0096 & 0.0001 & 0.0000 & 0.0000 & -0.0052 & 0.0000 \\
\hline
\end{tabular}




\begin{tabular}{|l|l|l|l|l|l|l|l|l|}
$\mathbf{S C}_{\mathbf{7}}$ & -0.0280 & 0.0008 & -0.0384 & 0.0015 & -0.0118 & 0.0001 & -0.0310 & 0.0010 \\
\hline $\mathbf{S C}_{\mathbf{8}}$ & 0.0000 & 0.0000 & -0.0479 & 0.0023 & 0.0000 & 0.0000 & 0.0000 & 0.0000 \\
\hline $\mathbf{S C} \mathbf{9}_{\mathbf{9}}$ & -0.0280 & 0.0008 & -0.0575 & 0.0033 & -0.0354 & 0.0013 & -0.0207 & 0.0004 \\
\hline $\mathbf{S C}_{\mathbf{1 0}}$ & -0.0466 & 0.0022 & -0.0192 & 0.0004 & -0.0236 & 0.0006 & -0.0155 & 0.0002 \\
\hline $\mathbf{S C}_{\mathbf{1 1}}$ & 0.0000 & 0.0000 & 0.0000 & 0.0000 & 0.0000 & 0.0000 & -0.0103 & 0.0001 \\
\hline $\mathbf{S C}_{\mathbf{1 2}}$ & -0.0373 & 0.0014 & -0.0384 & 0.0015 & -0.0472 & 0.0022 & -0.0362 & 0.0013 \\
\hline $\mathbf{S C}_{\mathbf{1 3}}$ & 0.0000 & 0.0000 & -0.0479 & 0.0023 & -0.0236 & 0.0006 & 0.0000 & 0.0000 \\
\hline $\mathbf{S C}_{\mathbf{1 4}}$ & -0.0093 & 0.0001 & -0.0384 & 0.0015 & 0.0000 & 0.0000 & -0.0207 & 0.0004 \\
\hline $\mathbf{S C}_{\mathbf{1 5}}$ & -0.0093 & 0.0001 & -0.0288 & 0.0008 & -0.0118 & 0.0001 & -0.0310 & 0.0010 \\
\hline $\mathbf{S C} \mathbf{1 6}$ & -0.0186 & 0.0003 & -0.0479 & 0.0023 & 0.0000 & 0.0000 & -0.0259 & 0.0007 \\
\hline $\mathbf{S C}_{\mathbf{1 7}}$ & -0.0186 & 0.0003 & -0.0575 & 0.0033 & 0.0000 & 0.0000 & -0.0310 & 0.0010 \\
\hline $\mathbf{S C}_{\mathbf{1 8}}$ & -0.0093 & 0.0001 & -0.0384 & 0.0015 & -0.0118 & 0.0001 & -0.0207 & 0.0004 \\
\hline
\end{tabular}

Step 5(b): The separation of each alternative from the negative ideal solution, $d_{i}^{-}$is

\begin{tabular}{|c|c|c|c|c|c|c|c|c|}
\hline \multirow{2}{*}{$\begin{array}{c}\text { Attribute } \\
\mathbf{S}\end{array}$} & \multicolumn{2}{|c|}{$\mathbf{C}_{\mathbf{1}}$} & \multicolumn{2}{|c|}{$\mathbf{C}_{\mathbf{2}}$} & \multicolumn{2}{c|}{$\mathbf{C}_{\mathbf{3}}$} & \multicolumn{2}{c|}{$\mathbf{C}_{\mathbf{4}}$} \\
\cline { 2 - 9 } & $v_{i j}-v_{j}^{-}$ & $\left(v_{i j}-v_{j}^{-}\right)^{2}$ & $v_{i j}-v_{j}^{-}$ & $\left(v_{i j}-v_{j}^{-}\right)^{2}$ & $v_{i j}-v_{j}^{-}$ & $\left(v_{i j}-v_{j}^{-}\right)^{2}$ & $v_{i j}-v_{j}^{-}$ & $\left(v_{i j}-v_{j}^{-}\right)^{2}$ \\
\hline $\mathbf{S C}_{\mathbf{1}}$ & 0.0466 & 0.0022 & 0.0479 & 0.0023 & 0.0472 & 0.0022 & 0.0259 & 0.0007 \\
\hline $\mathbf{S C}_{\mathbf{2}}$ & 0.0373 & 0.0014 & 0.0384 & 0.0015 & 0.0354 & 0.0013 & 0.0103 & 0.0001 \\
\hline $\mathbf{S C}_{\mathbf{3}}$ & 0.0373 & 0.0014 & -0.0288 & 0.0008 & 0.0118 & 0.0001 & 0.0000 & 0.0000 \\
\hline $\mathbf{S C}_{\mathbf{4}}$ & 0.0186 & 0.0003 & -0.0384 & 0.0015 & 0.0236 & 0.0006 & 0.0052 & 0.0000 \\
\hline $\mathbf{S C}_{\mathbf{5}}$ & 0.0373 & 0.0014 & 0.0288 & 0.0008 & 0.0472 & 0.0022 & 0.0155 & 0.0002 \\
\hline $\mathbf{S C}_{\mathbf{6}}$ & 0.0466 & 0.0022 & 0.0384 & 0.0015 & 0.0472 & 0.0022 & 0.0310 & 0.0010 \\
\hline $\mathbf{S C}_{\mathbf{7}}$ & 0.0186 & 0.0003 & -0.0096 & 0.0001 & 0.0354 & 0.0013 & 0.0052 & 0.0000 \\
\hline $\mathbf{S C}_{\mathbf{8}}$ & 0.0466 & 0.0022 & -0.0192 & 0.0004 & 0.0472 & 0.0022 & 0.0362 & 0.0013 \\
\hline $\mathbf{S C}_{\mathbf{9}}$ & 0.0186 & 0.0003 & 0.0000 & 0.0000 & 0.0118 & 0.0001 & 0.0155 & 0.0002 \\
\hline $\mathbf{S C}_{\mathbf{1 0}}$ & 0.0000 & 0.0000 & 0.0384 & 0.0015 & 0.0236 & 0.0006 & 0.0207 & 0.0004 \\
\hline $\mathbf{S C}_{\mathbf{1 1}}$ & 0.0466 & 0.0022 & 0.0767 & 0.0059 & 0.0472 & 0.0022 & 0.0259 & 0.0007 \\
\hline $\mathbf{S C}_{\mathbf{1 2}}$ & 0.0093 & 0.0001 & 0.0384 & 0.0015 & 0.0000 & 0.0000 & 0.0000 & 0.0000 \\
\hline $\mathbf{S C}_{\mathbf{1 3}}$ & 0.0466 & 0.0022 & 0.0288 & 0.0008 & 0.0236 & 0.0006 & 0.0362 & 0.0013 \\
\hline $\mathbf{S C}_{\mathbf{1 4}}$ & 0.0373 & 0.0014 & 0.0384 & 0.0015 & 0.0472 & 0.0022 & 0.0155 & 0.0002 \\
\hline $\mathbf{S C}_{\mathbf{1 5}}$ & 0.0373 & 0.0014 & 0.0479 & 0.0023 & 0.0354 & 0.0013 & 0.0052 & 0.0000 \\
\hline $\mathbf{S C}_{\mathbf{1 6}}$ & 0.0280 & 0.0008 & 0.0288 & 0.0008 & 0.0472 & 0.0022 & 0.0103 & 0.0001 \\
\hline $\mathbf{S C}_{\mathbf{1 7}}$ & 0.0280 & 0.0008 & 0.0192 & 0.0004 & 0.0472 & 0.0022 & 0.0052 & 0.0000 \\
\hline $\mathbf{S C}_{\mathbf{1 8}}$ & 0.0373 & 0.0014 & 0.0384 & 0.0015 & 0.0354 & 0.0013 & 0.0155 & 0.0002 \\
\hline
\end{tabular}


Step 5(c) and Step 6: The separation of each alternative from the positive $\left(d_{i}^{+}\right)$and negative $\left(d_{i}^{-}\right)$ ideal solutions are

\begin{tabular}{|c|c|c|c|c|c|}
\hline \multirow{2}{*}{ Attributes } & \multicolumn{2}{|c|}{ Positive Ideal Solution } & \multicolumn{2}{c|}{ Negative Ideal Solution } & \multirow{2}{*}{$D_{i}=\frac{d_{i}}{d_{i}^{+}}$} \\
\cline { 2 - 5 } & $\sum_{j=1}^{n}\left(v_{i j}-v_{j}^{+}\right)^{2}$ & $d_{i}^{+}=\sqrt{\sum_{j=1}^{n}\left(v_{i j}-v_{j}^{+}\right)^{2}}$ & $\sum_{j=1}^{n}\left(v_{i j}-v_{j}^{-}\right)^{2}$ & $d_{i}^{-}=\sqrt{\sum_{j=1}^{n}\left(v_{i j}-v_{j}^{-}\right)^{2}}$ & \\
\hline $\mathbf{S C}_{\mathbf{1}}$ & 0.0002 & 0.0141 & 0.0074 & 0.0858 & 0.0858 \\
\hline $\mathbf{S C}_{\mathbf{2}}$ & 0.0013 & 0.0355 & 0.0042 & 0.0650 & 0.0650 \\
\hline $\mathbf{S C}_{\mathbf{3}}$ & 0.0035 & 0.0590 & 0.0024 & 0.0485 & 0.0485 \\
\hline $\mathbf{S C}_{\mathbf{4}}$ & 0.0038 & 0.0614 & 0.0024 & 0.0490 & 0.0490 \\
\hline $\mathbf{S C}_{\mathbf{5}}$ & 0.0009 & 0.0297 & 0.0047 & 0.0685 & 0.0685 \\
\hline $\mathbf{S C}_{\mathbf{6}}$ & 0.0001 & 0.0109 & 0.0068 & 0.0827 & 0.0827 \\
\hline $\mathbf{S C}_{\mathbf{7}}$ & 0.0034 & 0.0579 & 0.0017 & 0.0415 & 0.0415 \\
\hline $\mathbf{S C}_{\mathbf{8}}$ & 0.0023 & 0.0479 & 0.0061 & 0.0780 & 0.0780 \\
\hline $\mathbf{S C}_{\mathbf{9}}$ & 0.0058 & 0.0760 & 0.0007 & 0.0270 & 0.0270 \\
\hline $\mathbf{S C}_{\mathbf{1 0}}$ & 0.0033 & 0.0578 & 0.0025 & 0.0496 & 0.0496 \\
\hline $\mathbf{S C}_{\mathbf{1 1}}$ & 0.0001 & 0.0103 & 0.0110 & 0.1047 & 0.9104 \\
\hline $\mathbf{S C}_{\mathbf{1 2}}$ & 0.0064 & 0.0800 & 0.0016 & 0.0395 & 0.0395 \\
\hline $\mathbf{S C}_{\mathbf{1 3}}$ & 0.0029 & 0.0534 & 0.0049 & 0.0698 & 0.0698 \\
\hline $\mathbf{S C}_{\mathbf{1 4}}$ & 0.0020 & 0.0446 & 0.0053 & 0.0730 & 0.0730 \\
\hline $\mathbf{S C}_{\mathbf{1 5}}$ & 0.0020 & 0.0449 & 0.0050 & 0.0705 & 0.0705 \\
\hline $\mathbf{S C}_{\mathbf{1 6}}$ & 0.0033 & 0.0576 & 0.0039 & 0.0628 & 0.0628 \\
\hline $\mathbf{S C}_{\mathbf{1 7}}$ & 0.0046 & 0.0680 & 0.0034 & 0.0584 & 0.0584 \\
\hline $\mathbf{S C}_{\mathbf{1 8}}$ & 0.0021 & 0.0461 & 0.0044 & 0.0660 & 0.0660 \\
\hline & & & & & \\
\hline
\end{tabular}

\section{Step 7:}

Rank the alternatives are $\mathrm{SC}_{11}>\mathrm{SC}_{1}>\mathrm{SC}_{6}>\mathrm{SC}_{8}>\mathrm{SC}_{14}>\mathrm{SC}_{15}>\mathrm{SC}_{13}>\mathrm{SC}_{5}>\mathrm{SC}_{18}>\mathrm{SC}_{2}>\mathrm{SC}_{16}>\mathrm{SC}_{17}>\mathrm{SC}_{10}>$ $\mathrm{SC}_{4}>\mathrm{SC}_{3}>\mathrm{SC}_{7}>\mathrm{SC}_{12}>\mathrm{SC}_{9}$ to the relative closeness to the ideal solution.

\section{Conclusion}

The paper applies a decision making method to rank the causes linked to the suicidal thought in domestic violence. Four criteria and eighteen alternatives were used to be analyzed using TOPSIS method. The method suggests that "He was unfaithful to you/had extra-marital relationships" is the main cause of our problem. This TOPSIS method give the ranking for the attributes, $0.9104>0.0858>0.0827>0.0780>0.0$ $730>0.0705>0.0698>0.0685>0.0660>0.0650>$ $0.0628>0.0584>0.0496>0.0490>0.0485>0.041$ $5>0.0395>0.0270$ i.e., the attributes, He was unfaithful to you/had extra-marital relationships, Did not permit to meet/interact with female, Accused you of being unfaithful to you, Treated you like a servant, Slapped you/Twisted you are/pulled your hair, Beat you on other body parts, Insulted you infront of others, Irritated/suspecious/angry if you talking to man, Attacked you with knife or some other weapon are highly promote the suicide thought in domestic violence.

\section{References}

[1] A.Awasthi, S.S Chauhan, S.K. Goyal, (2011), “A multi-critria decision making 
approach for location planning for urban distribution centres under uncertainty", Mathematical and Computer Modelling, 53(12) $98-109$.

[2] A.Victor Devadoss, M.Clement Joe Anand (2012), "A Solution to Control Suicide in the Domestic Violence using Combined Disjoint Block Fuzzy Cognitive Maps (CDBFCM)", International Journal of Scientific \& Engineering Research, Volume 3, Issue 6.Anderson and Roy (2010).

[3] B. Kosko (1988), "Fuzzy Cognitive Maps", International Journal of man-machine studies, January, 62-75.

[4] B. Kosko (1988) "Hidden patterns in combined and Adaptive Knowledge Networks", Proc. Of the First, IEEE International Conference on Neural Networks (ICNN-86377-393).

[5] B. Kosko (1997), "Neural Networks and Fuzzy systems: A Dynamical System Approach to Machine Intelligence", Prentice Hall of India.

[6] C.S Wu, C.T. Lin, C.Lee (2010), "Optimal marketing strategy: A decision-making with ANP and TOPSIS". International Journal of Production Economics, 127(1):190-196.

[7]C.P. Kiran, S. Clement, V.P Agrawal, (2011), "Coding evaluatin and optimal selection of a mechatronic system", Expert system with Application, 38(8): 9704-9712.

[8] G.A. Jordi, (2012), "Selection irrigatin water pricing alternatives using a multimethodological approach". Mathematical and Computer Modelling, 55(3-4): 861-883.

[9] George J.Klir/Bo Yuan (2010), "Fuzzy sets and Fuzzy Logic: Theory and Applications", Prentice Hall of India.

[10] G. Torlak, M. Sevkli, M. Sanal et al. (2011), "Analyzing business competition by using fuzzy TOPSIS method: An example of Turkish Domestic airline industry". Expert System with Application, 38(4): 3396-3406.
[11] J.S. Jia, Y. Fan, X.D. Guo, (2012), “The low carbon development (LCD) level's evaluation of the world's 47 countries(areas) by combining the FHP with the TOPSIS method", Expert System with Applications, 39(7): 6628-6640.

[12] Lbid.Ligon et. al (2004).Resolution $\mathrm{A} / \mathrm{RES} / 48 / 104$. "Declaration on the elimination of violence against women" adopted by the UN General Assembly, 20 December 1993.

[13] Response to deomestic violence in pregnancy- Report. Making preganancy safer and gender mainstreaming; 2005 April 12-13; The Republic of Moldova. Denmark: WHO Regional Office for Europe; p. 5-9, 2006.

[14] S.R. Schuler, S.M. Hashemi, A.P. riley and S. Akhter (1996), "Credit Programs, Partriarchy and Men's Violence against Women in Rural Bangaladesh", Soc. Sci Medicine Vol. 43, No.12, pp 1729-1742.

[15] T.Adel, H.M. Adel, (2011), “A group AHP-TOPSIS framwork for human spaceflight mission planning at NASA", Expert System with Applications, 38(11): 13588-13603.

[16]V. Patel (2005), "Gender in mental health research. In Gender and health research series", Geneva World-Health Organization; $p$. 11-21.

[17]S.Zhang, (2011), A model for evaluating computer network security systems with 2tuple linguistic information, Computers and Mathematics with Applications, 62(4): 19161922.

[18]Y.Ju, A. Wang, (2012), "Emergency alternative evaluation under group decision makers: A method of incorporating DS/AHP with extened TOPSIS", Expert System with Applications, 39(1): 1315-1323. 